\title{
IMAGE COMBINATION FOR HIGH-FIELD PHASED-ARRAY MRI*
}

\author{
Rui Yan ${ }^{1}$, Deniz Erdogmus ${ }^{1}$, Erik G. Larsson ${ }^{1}$, Jose C. Principe ${ }^{1}$, Jeffrey R. Fitzsimmons ${ }^{2}$ \\ ${ }^{1}$ Dept. of Electrical and Computer Eng., University of Florida, Gainesville, FL 32611, USA \\ ${ }^{2}$ Dept. of Radiology, University of Florida, Gainesville, FL 32610, USA
}

\begin{abstract}
We consider signal processing methods for phased-array magnetic resonance imaging (MRI). A theoretical description of a phasedarray MRI data model is presented and three image reconstruction algorithms are proposed to estimate the effective image pixels. An analysis is provided to show how the new algorithms compare to conventional reconstruction methods.
\end{abstract}

\section{INTRODUCTION}

The development of MRI was sparked in the early 1950's when Bloch and Purcell were awarded the Nobel Prize for their discovery of nuclear magnetic resonance. The idea of using multiple receiver coils, or phased-array coils, for MRI is about a decade old. The first implementation of a phased-array MR imaging system is probably due to Roemer et al., but the ideas of using multiple detectors for MRI can be traced back to the late 1980's. A good summary of this technology during the last ten years is provided in the review paper [1]. Recently, a substantial body of research has focussed on sophisticated techniques for phase encoding together with the use of gradient coils (with the primary aim of increasing the imaging speed). This work includes the sensitivity encoding for fast MRI (SENSE) technique [2] and Simultaneous Acquisition of Spatial Harmonics (SMASH) imaging [3]. Owing to the last decade's intensive research on the topic, phased-array MR imaging is now becoming a mature field and arrays with up to 16 elements have been designed and used for imaging experiments [4].

In principle, with phased-array technology, an increase in imaging speed equal to the number of parallel coils can be achieved. However, the use of large coil arrays imposes a number of difficulties, in particular for high field strengths. Most importantly, since the coil sensitivities are typically unknown variables (which are very difficult to model for high magnetic fields), optimal and artifact-free image reconstruction is a challenge. The most commonly used method for image reconstruction is the so-called "sum-of-squares" (SoS) method. However, it is not optimal; in particular, it introduces bias in the estimated image.

A number of somewhat more sophisticated techniques for image reconstruction with phased-array coils have appeared during the last decade. For example, as an alternative to the sum-ofsquares reconstruction, Debbins et al. [5] suggested to add the images coherently after their relative phase was properly adjusted. Bydder et al. [6] proposed a method that attempted to estimate the coil sensitivities from the image; the resulting image has somewhat less variance than the SoS reconstruction, however, it may still suffer from bias. Kellman and McVeigh [7] proposed a method that

${ }^{*}$ THIS WORK WAS PARTIALLY SUPPORTED BY THE NSF GRANT ECS-9900394. can use the degrees of freedom inherent to the phased array for ghost artifact cancellation. Another more sophisticated technique was presented by Walsh et al. [8] who used adaptive filters to improve the signal-to-noise ratio (SNR) in the image.

In this paper, we study the reconstruction problem from both least-squares and maximum-likelihood points of view. We also incorporate prior knowledge of the coil sensitivities via a Bayesian framework. This priori knowledge can obtained via, for example, calibration or electromagnetic modeling.

\section{THE PHASED-ARRAY MRI DATA MODEL}

A basic MRI signal model can be derived from the Bloch equation $[9,10]$. Let $\boldsymbol{x}, \boldsymbol{y}, \boldsymbol{z}$ be orthogonal unit vectors that span the Cartesian coordinate system under consideration, and suppose that a suitable gradient magnetic field is applied to enable selective excitation of a thin slice parallel to the $(\boldsymbol{x}, \boldsymbol{y})$-plane, say at $z=z_{0}$. At a given coordinate $x, y, z_{0}$ and time $t$, let $G_{x}(t)$ and $G_{y}(t)$ be the strength of the external magnetic field along the $x$ and $y$ axes and define

$$
k_{x}(t)=\int_{0}^{t} G_{x}(\tau) \mathrm{d} \tau, \quad \text { and } \quad k_{y}(t)=\int_{0}^{t} G_{y}(\tau) \mathrm{d} \tau
$$

Then, for a given receiver coil, the noise-free received timedomain signal can be written

$$
x(t)=e^{-i \omega_{0} t} \int_{x} \int_{y} \rho(x, y) C(x, y) e^{-i \lambda\left(k_{x}(t) x+k_{y}(t) y\right)} \mathrm{d} x \mathrm{~d} y
$$

where $\lambda$ is a constant, and $\rho(x, y)$ is proportional to the "transverse magnetization" (which is essentially the quantity of interest in the imaging), $C(x, y)$ is the sensitivity of the coil (i.e., the "antenna beampattern"). Eq. (2) shows that the received signal at time $t$ is essentially equal to the 2D Fourier transform of $C(x, y) \rho(x, y)$ evaluated at $k_{x}(t), k_{y}(t)$. Hence, by choosing an appropriate external time-varying magnetic field $G_{x}(t)$ and $G_{y}(t)$, the 2D Fourier transform of $C(x, y) \rho(x, y)$ can be sampled at several points $\left\{k_{x}, k_{y}\right\}$ and the MR image can be obtained via inverse Fourier transform.

In the phased-array MRI data model case, let $\rho$ be an $M$ vector that contains the image pixels of interest, suppose that the $k$-space is sampled at $N$ points and let us arrange these measured points in an $M$-vector $\boldsymbol{s}_{k}$ after the inverse Fourier transform. Also, let $\boldsymbol{c}_{k}$ be an $M$-vector of coil sensitivities associated with the $k$ th coil and the $M$ pixels. Let us assume that $\left\{c_{k}(n)\right\}$ is constant throughout a small region $\Omega$ consisting of $M$ pixels in the image, i.e., that

$$
c_{k}(n)=c_{k}
$$


For $n \in \Omega$ of interest, the data model is

$$
\boldsymbol{S}=\boldsymbol{\rho} \boldsymbol{c}^{T}+\boldsymbol{E}
$$

where $\boldsymbol{S}=\left[\begin{array}{lll}\boldsymbol{s}_{1} & \cdots & \boldsymbol{s}_{n_{c}}\end{array}\right]$ with $\boldsymbol{s}_{k}$ being the measurement of the $k^{\text {th }}$ coil, and $\boldsymbol{E}$ is a "local" noise which is zero-mean, independent between two different points in space domain, but possibly correlated between the receiver coils. For known $\boldsymbol{c}, \boldsymbol{\rho}$ in (4) can be determined via least-squares, whereas for unknown $\boldsymbol{c}$, the problem becomes more complicated.

\section{METHODS}

\subsection{Sum-of-Squares (SoS) Reconstruction}

With the SoS method, the data is first pre-whitened by $\boldsymbol{Q}^{-1 / 2}$ to account for the noise coloration, and thereafter each measured pixel is taken as an estimate of the coil sensitivity. For simplicity, we neglect the noise coloration $\boldsymbol{Q}$ in our description. Then the reconstructed pixel is obtained via

$$
\hat{\boldsymbol{\rho}}(n)=\sqrt{\sum_{k=1}^{n_{c}}\left|\boldsymbol{s}_{k}(n)\right|^{2}}
$$

Clearly, if the noise level goes to zero the SoS-estimate converges to $\hat{\boldsymbol{\rho}}(n)=\sqrt{\sum_{k=1}^{n_{c}}\left|\boldsymbol{c}_{k}(n) \boldsymbol{\rho}(n)\right|^{2}}$ which is in general not equal to $\boldsymbol{\rho}(n)$. Therefore, SoS reconstruction yields in general severely biased reconstructions, even in the noise-free case. Unless $\boldsymbol{c}(n)$ is constant throughout the image (which is certainly not the case in practice), this bias depends on the pixel number $n$ and hence it cannot be corrected for if $\boldsymbol{c}(n)$ is unknown.

\subsection{Singular Value Decomposition Reconstruction}

Singular value decomposition (SVD) is widely implemented in signal processing applications. In ideal noise-free environment, the left singular vector and the right singular vector of $\boldsymbol{S}$ is the desired pixel vector $\boldsymbol{\rho}$ and the coil sensitivity vector $\boldsymbol{c}$. However, the introduction of noise increases the rank of the matrix $\boldsymbol{S}$ to greater than one, and hence the left singular vector and the right singular vector corresponding to the maximum singular value will yield the least-squares estimate of $\boldsymbol{\rho}$ and $\boldsymbol{c}$. In the case of $\operatorname{rank}(\boldsymbol{S})=n_{c}$, the pixel vector is estimated as

$$
\hat{\boldsymbol{\rho}}=\boldsymbol{u}_{1}
$$

where

$$
\boldsymbol{S}=\underbrace{\left[\begin{array}{lll}
\boldsymbol{u}_{1} & \cdots & \boldsymbol{u}_{n_{c}}
\end{array}\right]}_{\boldsymbol{U}} \underbrace{\left[\begin{array}{ccc}
\lambda_{1} & \cdots & 0 \\
\vdots & \ddots & \vdots \\
0 & \cdots & \lambda_{n_{c}}
\end{array}\right]}_{\Sigma} \underbrace{\left[\begin{array}{lll}
\boldsymbol{v}_{1} & \cdots & \boldsymbol{v}_{n_{c}}
\end{array}\right]}_{\boldsymbol{V}},
$$

and $\lambda_{1} \geq \lambda_{2} \geq \cdots \geq \lambda_{n_{c}}, \boldsymbol{u}_{k}$ is the $M$ dimensional left singular vector of $\boldsymbol{S}$; furthermore, $\boldsymbol{v}_{k}$ is the $n_{c}$ dimensional right singular vector $\left(k=1, \cdots, n_{c}\right)$. There is a scaling ambiguity in the sense that no unique $\hat{\rho}$ satisfies the decomposition. For instance, we can use the norm of the SoS solution to scale $\hat{\rho}$ to recover the image contrast in different local regions.

\subsection{Coil-Average Reconstruction}

The key problem to extract $\boldsymbol{\rho}$ from each column of signal $\boldsymbol{s}_{k}$ is to "cancel" the coil sensitivity $\boldsymbol{c}_{k}$. The local constancy of $\boldsymbol{c}_{k}$ connotes $\boldsymbol{s}_{k}=\boldsymbol{c}_{k} \boldsymbol{\rho}, \quad k=1, \cdots, n_{c}$. Therefore the normalization of $\boldsymbol{s}_{k}$ eliminates the coil sensitivity $\boldsymbol{c}_{k}$ in each coil, and the $\boldsymbol{s}_{k}$ average gives the normalized $\hat{\boldsymbol{\rho}}$ via

$$
\hat{\boldsymbol{\rho}}=\frac{1}{n_{c}} \sum_{k=1}^{n_{c}} \frac{\boldsymbol{s}_{k}}{\left\|\boldsymbol{s}_{k}\right\|}
$$

For this method, the SoS norm is also used to normalize $\hat{\boldsymbol{\rho}}$.

\subsection{Bayesian Reconstruction}

Under the assumptions made on $\boldsymbol{E}$, the columns of the matrix $\boldsymbol{S}$ are (conditioned on $\boldsymbol{\rho}$ and $\boldsymbol{c}$ ) independent and circularly symmetric Gaussian with mean $\boldsymbol{\rho} \boldsymbol{c}^{T}$ and covariance $\boldsymbol{Q}$. Hence the p.d.f. of $\boldsymbol{S}$ is equal to

$$
p(\boldsymbol{S} \mid \boldsymbol{\rho}, \boldsymbol{c})=\pi^{-M n_{c}}|\boldsymbol{Q}|^{-n_{c}} \exp \left(-\left\|\left(\boldsymbol{S}-\boldsymbol{\rho} \boldsymbol{c}^{T}\right) \boldsymbol{Q}^{-1 / 2}\right\|^{2}\right)
$$

Suppose that the prior knowledge of the coil sensitivities $\boldsymbol{c}$ can be described by assuming that $\boldsymbol{c}$ is Gaussian with mean $\boldsymbol{\mu}$ and covariance $\boldsymbol{\Lambda}$. Then the p.d.f. of $\boldsymbol{c}$ is given by

$$
p(\boldsymbol{c})=\pi^{-n_{c}}|\boldsymbol{\Lambda}|^{-1} \exp \left(-\left\|\boldsymbol{\Lambda}^{-1 / 2}(\boldsymbol{c}-\boldsymbol{\mu})\right\|^{2}\right)
$$

From Bayesian theorem, the p.d.f of $\boldsymbol{S}$ is expressed as

$$
\begin{aligned}
& p(\boldsymbol{S} \mid \boldsymbol{\rho})=\int p(\boldsymbol{S}, \boldsymbol{c} \mid \boldsymbol{\rho}) \mathrm{d} \boldsymbol{c}=\int p(\boldsymbol{S} \mid \boldsymbol{\rho}, \boldsymbol{c}) p(\boldsymbol{c}) \mathrm{d} \boldsymbol{c} \\
& =\int \pi^{-n_{c}}|\boldsymbol{\Lambda}|^{-1} \exp \left(-\left\|\boldsymbol{\Lambda}^{-1 / 2}(\boldsymbol{c}-\boldsymbol{\mu})\right\|^{2}\right) \\
& \cdot \pi^{-M n_{c}}|\boldsymbol{Q}|^{-n_{c}} \exp \left(-\left\|\left(\boldsymbol{S}-\boldsymbol{\rho} \boldsymbol{c}^{T}\right) \boldsymbol{Q}^{-1 / 2}\right\|^{2}\right) \mathrm{d} \boldsymbol{c} \\
& =\pi^{-(M+1) n_{c}}|\boldsymbol{Q}|^{-n_{c}}|\boldsymbol{\Lambda}|^{-1} \\
& \int \exp \left(-\left\|\left(\boldsymbol{S}-\boldsymbol{\rho} \boldsymbol{c}^{T}\right) \boldsymbol{Q}^{-1 / 2}\right\|^{2}-\left\|\boldsymbol{\Lambda}^{-1 / 2}(\boldsymbol{c}-\boldsymbol{\mu})\right\|^{2}\right) \mathrm{d} \boldsymbol{c}
\end{aligned}
$$

The incorporation of a priori knowledge of model parameters via Bayesian statistics has a number of the distinct advantages. For example, the degree of uncertainty about $c$ can be easily introduced as a model parameter $\boldsymbol{\Lambda}$. In the extreme case that there is a complete lack of a priori information about $\boldsymbol{c}$, we can take $\boldsymbol{\mu}$ arbitrary and $\boldsymbol{\Lambda}$ very large (i.e., letting it grow without bound). In the other extreme case when we have full knowledge of the parameter $\boldsymbol{c}$, we can simply take $\boldsymbol{\mu}$ equal to its known value and set $\boldsymbol{\Lambda}=\mathbf{0}$ (in this case, the p.d.f. in (9) effectively reduces to a Dirac-like impulse function).

The above integral result is a product of an exponential function multiplied with an determinant, where both have a $\boldsymbol{Q}^{-\frac{T}{2}} \otimes \boldsymbol{\rho}$ part. It appears not to be directly straightforward to maximize the total p.d.f. with respect to $\boldsymbol{\rho}$, and as an approximation we simply minimize the sum of the two norms inside the integral in (10) with respect to both the parameters $\boldsymbol{\rho}$ and $\boldsymbol{c}$. For this purpose, we use a 
cyclic algorithm:

1. begin initialize $\rho_{0}, T, i=0$

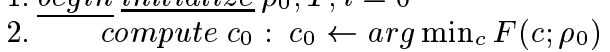

3. $\underline{d o} i \leftarrow i+1$

4. compute $\rho: \rho_{i+1} \leftarrow \arg \min _{\rho} F\left(\rho ; c_{i}\right)$

4. $\quad c_{i+1} \leftarrow \arg \min _{c} F\left(c ; \rho_{i}\right)$

5. until $F\left(\rho_{i+1}, c_{i+1}\right)-F\left(\rho_{i}, c_{i}\right)<T$

6. $\underline{\text { return }} \hat{\rho} \leftarrow \rho_{i+1}, \hat{c} \leftarrow c_{i+1}$

7. end

where the cost function $F$, the pixel vector $\rho$ and the coil sensitivity $\boldsymbol{c}$ are related by

$$
\begin{gathered}
F=\left\|\boldsymbol{\Lambda}^{-1 / 2}(\boldsymbol{c}-\boldsymbol{\mu})\right\|^{2}+\left\|\left(\boldsymbol{S}-\boldsymbol{\rho} \boldsymbol{c}^{T}\right) \boldsymbol{Q}^{-1 / 2}\right\|^{2} \\
\boldsymbol{c}_{i+1}=\left[\boldsymbol{T}_{i}^{H} \boldsymbol{T}_{i}+\boldsymbol{\Lambda}^{-\frac{H}{2}} \boldsymbol{\Lambda}^{-\frac{1}{2}}\right]^{-1}\left[\boldsymbol{T}_{i}^{H} \tilde{\boldsymbol{S}}+\boldsymbol{\Lambda}^{-\frac{H}{2}} \tilde{\boldsymbol{\mu}}_{c}\right] \\
\boldsymbol{\rho}_{i+1}=\left[\boldsymbol{B}_{i}^{H} \boldsymbol{B}_{i}\right]^{-1} \boldsymbol{B}_{i}^{H} \tilde{\boldsymbol{S}}
\end{gathered}
$$

where $\otimes$ stands for the Kronecker product and

$$
\begin{aligned}
& \tilde{\boldsymbol{B}}_{i}=\left(\boldsymbol{Q}^{-\frac{T}{2}} \boldsymbol{c}_{i}\right) \otimes \boldsymbol{I}, \quad \tilde{\boldsymbol{S}}=\left(\boldsymbol{Q}^{-\frac{T}{2}} \otimes \boldsymbol{I}\right)\left[\begin{array}{c}
\boldsymbol{s}_{1} \\
\vdots \\
\boldsymbol{s}_{n_{c}}
\end{array}\right] \\
& \tilde{\boldsymbol{T}}_{i}=\left(\boldsymbol{Q}^{-\frac{T}{2}}\right) \otimes \boldsymbol{\rho}_{i}, \quad \tilde{\boldsymbol{\mu}}_{c}=\boldsymbol{\Lambda}^{-\frac{1}{2}} \boldsymbol{\mu}_{c}
\end{aligned}
$$

\section{NUMERICAL RESULTS}

In order to compare the performance of the above four algorithms, we assume for simplicity that the pixels $\boldsymbol{\rho}$ in each column of $\boldsymbol{s}_{k}$ have the same SNR

$$
\boldsymbol{s}_{k}=\boldsymbol{c}_{k}\left(\boldsymbol{\rho}+\boldsymbol{e}_{k}\right)
$$

where $\boldsymbol{e}_{k}$ is the zero-mean, independent Gaussian noise with the same covariance matrix for the different coils.

Example 1 : The MRI signals detected by four coils with known varied coil sensitivities in a local region are simulated. The signal-to-error-ratio (SER) and signal-to-noise-ratio (SNR) are defined as

$$
\begin{aligned}
\boldsymbol{S E R} & \triangleq 10 \log _{10} \frac{E\left[\boldsymbol{\rho}^{T} \boldsymbol{\rho}\right]}{E\left[(\hat{\boldsymbol{\rho}}-\boldsymbol{\rho})^{T}(\hat{\boldsymbol{\rho}}-\boldsymbol{\rho})\right]} \\
\boldsymbol{S N R} & \triangleq 10 \log _{10} \frac{E\left[\boldsymbol{\rho}^{T} \boldsymbol{\rho}\right]}{\boldsymbol{\sigma}_{e}^{2}}
\end{aligned}
$$

where $\boldsymbol{\rho}$ and $\hat{\boldsymbol{\rho}}$ denote the true pixel sequence and the estimated one with $\|\boldsymbol{\rho}\|=1$ and $\|\hat{\boldsymbol{\rho}}\|=1$, and $\boldsymbol{\sigma}_{e}^{2}$ denotes the identical noise variance in each coil. By using simulated data we can construct a fully controlled experiment. Fig. 1 shows the SER against the SNR for the four algorithms in this specified region. SoS has relatively worse SER compared to the other three because it introduces positive bias to the estimated pixels. The SVD method has a gain of $7 \mathrm{~dB}$ on SER compared to SoS when SNR is 10dB. However, SVD is worse than SoS when the SNR is less than $4 \mathrm{~dB}$. It shows that $\hat{\rho}$ is sensitive to the noise interference. The CoilAverage algorithm has a general 12dB SER gain beyond SoS, because average itself means a gain of SER. In Bayesian Reconstruction algorithm, we choose the SoS solution as the initial pixel estimate $\hat{\boldsymbol{\rho}}$ and the coil sensitivity estimate $\hat{\boldsymbol{c}}$ in the first iteration as the $\boldsymbol{\mu}$ and the identity matrix as $\boldsymbol{\Lambda}$. It demonstrates the highest SER among the four algorithms. The SER is $7 \mathrm{~dB}$ and $15 \mathrm{~dB}$ higher than

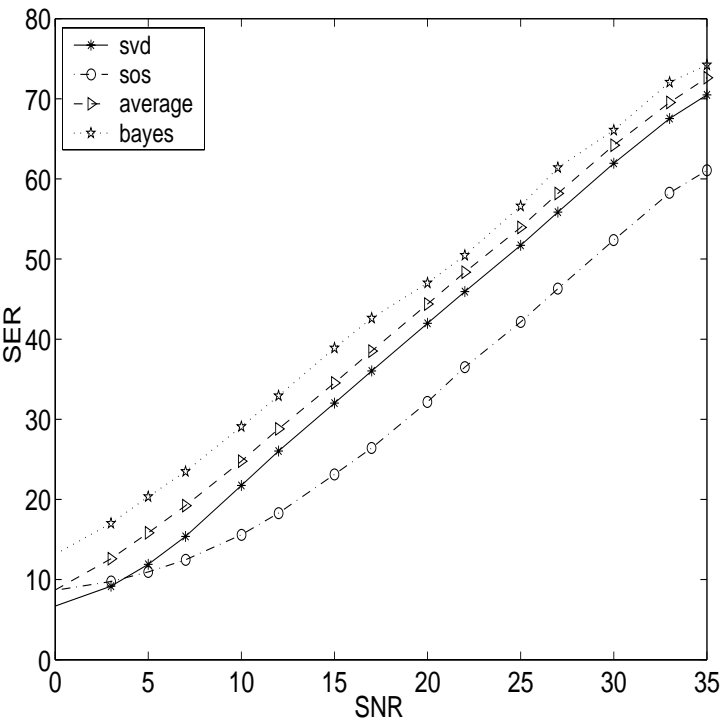

Fig. 1. Signal-to-error-ratio (SER) of the Bayesian, SVD, CoilAverage, and SoS, along signal-to-noise-ratio (SNR).

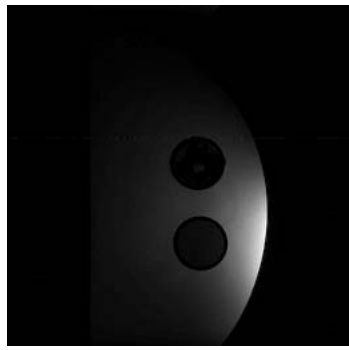

(a) half moon cylinder phantom.

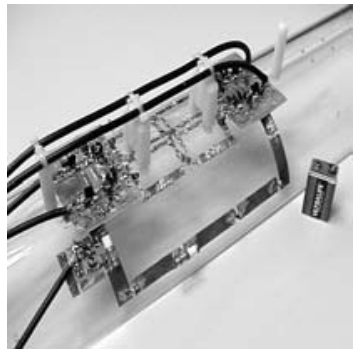

(b) Photograph of phased array coil, transmit coil, and cabling.
Fig. 2. Phantom image and its experimental devices.

SoS with SNR at $0 \mathrm{~dB}$ and $15 \mathrm{~dB}$, respectively. It is because of the prior information of coil sensitivity, the recursive approach and the good initial estimate that the Bayesian algorithm is promising.

Example 2 : For real data, the signal model in (4) is justified by the experimental phantom data in Fig. 2(a) (only one coil is presented due to the space limitation). It consists of a cat spinal cord in one pipe and another oil-filled pipe for reference, both inside a water-filled cylinder. The data is collected by a four-coil phased array $(\mathrm{TR}=1000 \mathrm{~ms}, \mathrm{TE}=15 \mathrm{~ms}, \mathrm{FOV}=10 \times 5 \mathrm{~cm}$, matrix $=256 \times 128$, slice thickness $=2 \mathrm{~mm}$, sweep width $=26 \mathrm{khz}, 1$ average) shown in Fig. 2(b) [11]. Fig. 3 shows the histogram of the ratio of the maximum singular value to the mean of the smaller three for the phantom data. In noise-free environment, all ratio values should be infinite. In the real phantom data case, most ratios range from 30 to 120 showing that the singularity assumption of the signal matrix $\boldsymbol{S}$ is well satisfied within reasonable numerical accuracy.

Example 3: We also use our algorithms on data from a 


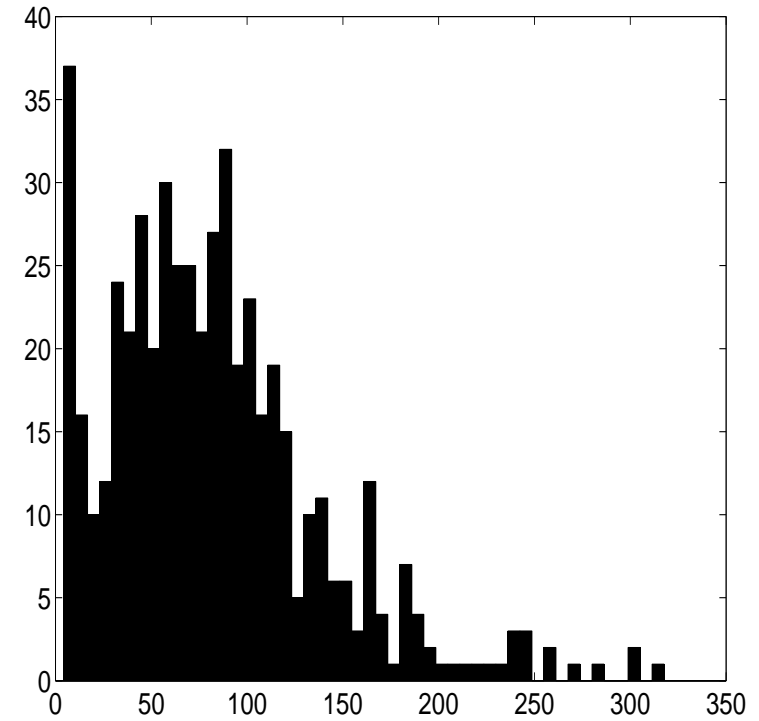

Fig. 3. Histogram of singular value ratio for phantom data.

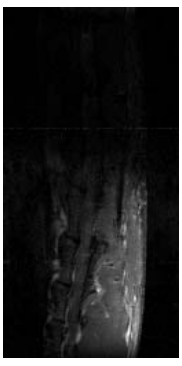

(a) Coil 1

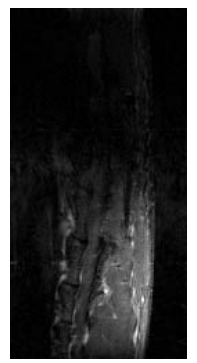

(b) Coil 2

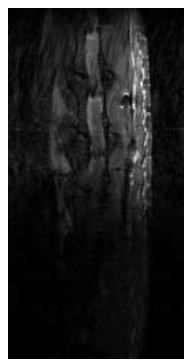

(c) Coil 3

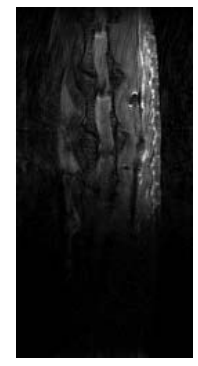

(d) Coil 4
Fig. 4. Vivo sagittal images of a cat spinal cord from four coils.

cat spinal cord with the same devices used in phantom data. Fig. 4 shows the images from four coils before reconstruction and Fig. 5 shows the reconstructed results by the four algorithms. In this case we do not pursue statistical analysis of the images due to the difficulties associated with finding both a proper reference image and the valuable image parts to judge the image quality.

\section{CONCLUDING REMARKS}

In summary, a data model for the multiple phased-array coils is set up based on the assumption of the local constancy of coil sensitivities. The model is justified by experimental data. Three new image reconstruction methods, namely SVD Reconstruction, CoilAverage Reconstruction and Bayesian Reconstruction, are compared to SoS. A computer simulation was implemented to compare the SER against the SNR of the above four methods. The Bayesian method indicates the highest SER while SoS shows the worst one using a known simulated true image. Further work is needed and will be performed in order to improve the performance of the methods.

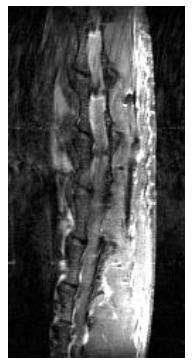

(a)

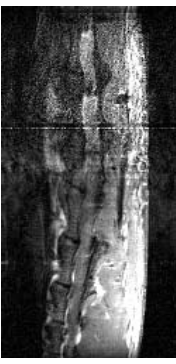

(b)

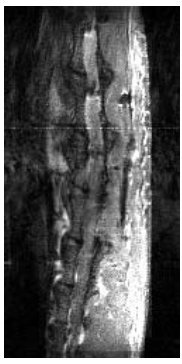

(c)

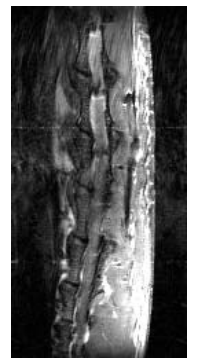

(d)
Fig. 5. Reconstruction images of cat spinal cord by methods of (a) Bayesian, (b) SVD, (c) Coil-Average and (d) SoS.

\section{REFERENCES}

[1] S. M. Wright and L. L. Wald, "Theory and application of array coils in MR spectroscopy," NMR in Biomedicine, vol. 10, pp. 394-410, 1997.

[2] K. P. Pruessmann, M. Weiger, M. B. Scheidegger, and P. Boesiger, "SENSE: sensitivity encoding for fast MRI," Magnetic Resonance in Medicine, vol. 42, pp. 952-962, 1999.

[3] J. A. Bankson, M. A. Griswold, S. M. Wright, and D. K. Sodickson, "SMASH imaging with an eight element multiplexed RF coil array," Magnetic Resonance Materials in Physics, Biology and Medicine (MAGMA), vol. 10, pp. 93-104, 2000.

[4] J. R. Porter, S. M. Wright, and A. Reykowski, "A 16-element phased-array head coil," Magnetic Resonance in Medicine, vol. 40, pp. 272-279, 1998.

[5] J. P. Debbins, J. P. Felmlee, and S. J. Riederer, "Phase alignment of multiple surface coil data for reduced bandwidth and reconstruction requirements," Magnetic Resonance in Medicine, vol. 38, pp. 1003-1011, 1997.

[6] M. Bydder, D. J. Larkman, and J. V. Hajnal, "Combination of signals from array coils using image-based estimation of coil sensitivity profiles," Magnetic Resonance in Medicine, vol. 47, pp. 539-548, 2002.

[7] P. Kellman and E. R. McVeigh, "Ghost artifact cancellation using phased array processing," Magnetic Resonance in Medicine, vol. 46, pp. 335-343, 2001.

[8] D. O. Walsh, A. F. Gmitro, and M. W. Marcellin, "Adaptive reconstruction of phased array MR imagery," Magnetic Resonance in Medicine, vol. 43, pp. 682-690, 2000.

[9] E. M. Haacke, R. W. Brown, M. R. Thompson, and R. Venkatesan, Magnetic Resonance Imaging - Physical Principles and Sequence Design. New York, NY: John Wiley and Sons, Inc., 1999.

[10] G. A. Wright, "Magnetic resonance imaging," IEEE Signal Processing Magazine, pp. 56-66, Jan. 1997.

[11] B. L. Beck and S. J. Blackband, "Phased array imaging on a $4.7 \mathrm{~T} / 33 \mathrm{~cm}$ animal research system," Review of Scientific Instruments, vol. 72, no. 11, pp. 4292-4294, 2001. 